\title{
Sit-to-stand movement score and locomo-age to detect locomotive syndrome in older adults
}

Go Yamako ( $\sim$ g.yamako@cc.miyazaki-u.ac.jp )

University of Miyazaki

Niroshan G. Punchihewa

University of Miyazaki

Hideki Arakawa

University of Miyazaki

Takuya Tajima

University of Miyazaki

Etsuo Chosa

University of Miyazaki

\section{Research Article}

Keywords: sit to stand, balance, mobility, aging, locomotive syndrome

Posted Date: July 31st, 2021

DOl: https://doi.org/10.21203/rs.3.rs-677930/v1

License: (c) (1) This work is licensed under a Creative Commons Attribution 4.0 International License.

Read Full License 


\section{Abstract}

Background Aging is characterized by biological changes, such as reductions in muscle strength and the loss of mobility and balance. The Japanese Orthopaedic Association proposed the term "locomotive syndrome" (LS) to define people at risk of requiring nursing care because of problems with locomotive system. A need exists to develop simple methods to quantify motor performance and allow the early detection of LS. Sit-to-stand (STS) movements are a mechanically demanding daily task and represent motor performance. Using the Nintendo Wii Balance Board (WBB), we have developed a novel method to quantify STS movements-the "STS score." This study investigated differences in the STS score across LS severity in healthy older adults. To recognize motor performance easily, we then defined the "locomoage" to represent the individual's age based on the LS status.

Methods We investigated whether the STS score can predict LS. This study enrolled 152 participants of 65 years and above. Participants were divided into three groups based on LS risk test results. Participants were instructed to stand up as quickly as possible on the WBB, and the STS score was calculated based on the combination of the speed and balance indices. We also evaluated motor status by calculating the peak power during STS and performing a timed up and go test and handgrip strength test. To calculate locomo-age, we used a regression equation between STS scores and age from a data set in our previous study.

Results The LS stage was increased significantly with age, whereas the association with body mass index was not significant. The STS score and timed up and go results were good indicators to detect LS. However, neither peak power nor handgrip strength could significantly distinguish between the LS stages.

Conclusions Measurement of the LS is important to assess older adults' motor performance. The novel STS score is an easy-to-use method that requires limited space and only one person to operate. The STS score decreased significantly with the LS severity and thus could be used to assess LS in older adults. The locomo-age of the STS score is useful to understand the LS status.

\section{Background}

Aging is characterized by biological changes, such as reductions in muscle mass and strength and the loss of mobility and balance [1]. This physiological deterioration leads to difficulties in performing activities of daily living (ADLs), and consequently, a decrease in the quality of life (QOL). In 2007, the aging rate in Japan exceeded $21 \%$, and the country is now facing a "super-aged" society. The Japanese Orthopaedic Association (JOA) thus proposed a new term-"locomotive syndrome" (LS)-to define individuals at risk of requiring nursing care services because of difficulties in living independently as a consequence of problems in locomotive organs, such as muscles, bones, and joints [2]. To improve the QOL of older adults, the JOA promotes physical exercise training and developed a risk test for LS based on the 25-question Geriatric Locomotive Function Scale (25-question GLFS), two-step test, and stand-up test [3]. The LS risk test is now used to screen a decline in motor performance [4]. However, this test is 
time consuming (more than 5 minutes per test-taker), and it requires a large space (more than $12 \mathrm{~m}^{2}$ ) and sufficient staff (more than 3 individuals) to perform the test safely. The 25-question need a long time to complete and the response rate is low in older people [5]. For most clinics, these requirements limit the use of the LS risk test on a daily basis. These issues motivated us to develop new methods to easily and quantitatively evaluate motor performance to identify the decline in mobility and LS.

We have thus developed a new method to evaluate motor performance based on sit-to-stand (STS) movement [6]. The STS movement is quantified by the STS score, defined as a combination of the speed and balance parameters using the vertical ground reaction force (VGRF) and center of pressure (COP) data measured from the Nintendo Wii Balance Board (WBB; Nintendo, Kyoto, Japan). Our previous study demonstrated that the STS score can detect age-related deterioration in motor performance based on data from 503 healthy individuals aged 20-88 years, without musculoskeletal or neuromuscular disorders [6]. In ADLs, STS movements are important tasks; a decreased ability to perform this movement is associated with falling [7], hip fracture [8], and institutionalization, or even death [9]. In addition, the STS movement has been identified as mechanically demanding because this action requires significant muscle strength, a wide joint movement, and good balance control [10, 11]. Accordingly, age-related decreases in muscle strength and balance control are associated frequently with difficulties in completing STS movements [12-14]. Thus, STS movement has been identified as a valuable source of an individual's motor performance [14-18].

We thus hypothesized that the STS score can predict the LS status. This study investigated differences in the STS score across the range of severity of LS in healthy older adults. In addition, we evaluated whether the performance of the timed up and go (TUG) test and the handgrip strength test could predict the LS status. Finally, we defined "locomo-age" as an individual's age represented by the LS status based on the regression curve of the STS score.

\section{Methods}

\section{Participants}

A total of 152 healthy participants older than 65 years (age range, 65-88 years; male/female ratio, 59:93) were recruited from local senior citizen clubs (called rojin clubs in Japanese). None of the participants had any known musculoskeletal or neuromuscular conditions that would limit their mobility or their ability to perform the STS movement. Research protocol of this study followed the Declaration of Helsinki. The ethical committee of our university approved the study protocol (reference number: 2014231), and all participants provided written informed consent.

\section{LS Risk Test to Classify LS Grade}

The participants were categorized into three groups according to the severity of LS, determined by the LS risk test $[3,19]$. The LS risk test consisted of three parts: (1) the 25-question GLFS, (2) the two-step test, and (3) the stand-up test. 


\section{(1) The 25-Question GLFS}

This scale is a self-administered, comprehensive measure, consisting of 25 items that include 4 questions on pain, 16 questions on ADLs, 3 questions on social functions, and 2 questions on mental health status. Each question is graded with a 5-point scale, from no impairment ( 0 points) to severe impairment (4 points). Participants are scored for a total by adding the points for each question (minimum $=0$; maximum $=100$ points). Thus, a higher score is associated with decreased mobility performance.

\section{(2) Two-Step Test}

This test measures the stride length to assess walking ability, including muscle strength, balance, and flexibility of the lower limbs. The procedure is as follows. Participants were instructed to take two long steps, as long as possible, and then to align both feet. The score is calculated by normalizing the maximal length of the two steps taken by the participant based on the participant's height.

\section{(3) Stand-Up Test}

This test assesses leg strength. Participants stand up on one leg or both legs from decreasing a chair height from 40 to $10 \mathrm{~cm}$, with a $10 \mathrm{~cm}$ stepdown. Lowering the chair height leads to a greater biomechanical demand while standing up from a seated position. Participants were instructed to stand up on one leg or both legs from each chair height without leaning back and then maintain the standing posture for 3 seconds. They were scored based on their height level using one leg and both legs. Scores ranged from 0 to 8 depending on their difficulty in standing up [4].

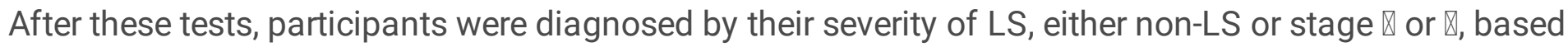
on the JOA protocol [20]. Stage $\mathbb{L S}$ represents the beginning of the decline in mobility. An individual who cannot perform a one-leg stand-up movement from a seat $40 \mathrm{~cm}$ high, or whose 25-question GLFS score is $\geq 7$, or whose two-step test score is $<1.3$ is diagnosed with stage $\nabla$ LS. Stage $\nabla$ LS represents progression to a decline in mobility. An individual who cannot perform a both-leg stand-up movement from a seat $20 \mathrm{~cm}$ high, or whose 25 -question GLFS score is $\geq 16$, or whose two-step test score is $<1.1$ is diagnosed with stage $₫$.

\section{STS Score}

Details of the STS testing procedure were described in our previous study [6]. Participants were instructed to stand up from a chair as quickly as possible, immediately recover their balance, and stand as still as possible in an upright posture for 5 seconds. To perform this test, participants were seated on an armless, backless chair, with the seat height adjusted to $100 \%$ of the participant's knee height (Fig. 1). The participants crossed their arms over their chest during testing. Both feet were placed shoulder-width apart on the WBB that was used as a force plate. The WBB is designed to serve as a video game controller that is increasingly used to assess postural control in rehabilitation [21-26]. The WBB, which consists of a rigid platform with four strain gauge-based vertical-load transducers located in the feet at each corner, 
was used to calculate the VGRF and COP. During each STS movement, WBB data were streamed to a laptop computer at approximately $100 \mathrm{~Hz}$. Before data were recorded, each participant was allowed the opportunity to practice the procedure. Each participant performed two trials, separated by a 1 minute interval. We did not observe any accidents, such as falling, during this test.

The STS score, which represents motor performance, was defined as a combination of the speed and balance scores. These parameters were calculated using the VGRF (in N), COP position in X and $\mathrm{Y}$ directions ( $\mathrm{Cx}, \mathrm{Cy}$; in $\mathrm{cm}$ ), and COP trajectory distance (in $\mathrm{cm}$ ) during STS movement (Fig. 2). The VGRF was normalized to the participant's body weight. The speed score $\left(\mathrm{s}^{-1}\right)$ was defined as a linear slope of the VGRF-time curve. The balance score $\left(\mathrm{m}^{-1}\right)$ was defined as the inverse of COP trajectory distance when Cy was minimized to +3 seconds. The STS score $\left((\mathrm{ms})^{-1}\right)$ was then defined as the product of the speed and balance scores to quantify STS movement performance. Higher STS scores indicated better motor performance. These two indices have a tradeoff relationship because typically if the movement speed (speed score) increases, it becomes difficult to control balance and remain as still as possible (balance score).

Indices were calculated from each set of trial data using a custom MATLAB program (MathWorks, Natick, $\mathrm{RI}$, USA). For each participant, a practice trial was followed by two timed trials, and the average value was used as a result for further analysis.

\section{Peak Power during STS}

We all calculated the peak power during the STS movement using the WBB data. All ADLs require the body's center of mass to move from one place to another by producing adequate force from the muscles. Explosive power is proven to be a good indicator to identify the functional ability of aging people [27]. The rising phase during STS was detected based on the VGRF [28]. Power during this period was calculated by integrating the acceleration derived by the VGRF (velocity) followed by taking the scalar product of the force and velocity [29].

\section{TUG Time}

The TUG test, a well-known clinical test, was developed to improve evaluations of functional performance and mobility [30]. This test measured the time needed for a participant to rise from a chair, walk $3 \mathrm{~m}$, turn around, walk back, turn around, and sit down again. A shorter TUG time indicates better performance. Participants were instructed to walk as quickly and safely as possible. For each participant, a practice trial was followed by two timed trials, and the fastest trial was selected for further analysis.

\section{Handgrip Strength}

Handgrip strength is a good index to positively correlate with motor function and ADL performance [31]. The strength was measured bilaterally in a standing position using a handgrip dynamometer. Both hands were tested two times, and the maximum value was used to characterize the participant's handgrip strength. 


\section{Statistical Analyses}

Statistical analyses were performed using SPSS 22.0 (IBM SPSS, Chicago, IL, USA). One-way analysis of variance and the Dunnett T3 multiple comparison test were used to analyze the differences among the three groups, as defined by the severity of LS (non-LS, stage $\otimes$ LS, stage $\otimes L S$ ). A $p$ value of $<0.05$ was considered statistically significant.

\section{Results}

\section{Baseline Characteristics of Enrolled Participants}

Among the 152 participants, 120 participants (78.9\%) were diagnosed with LS (Table 1). The percentage of participants with non-LS was $19.8 \%$, stage $\otimes$ LS was $42.1 \%$, and stage $\otimes$ LS was $38.1 \%$. The severity of LS increased significantly with age among participants $(p<0.001)$. No significant difference was noted in the body mass index among the three groups $(p=0.296)$. The results of the LS risk test, including the 25question GLFS, two-step test, and stand-up test, are shown in Table 1.

Table 1

Demographic Data and LS Risk Test Scores for Each LS Stage

\begin{tabular}{|c|c|c|c|}
\hline Demographic Data & Non-LS & 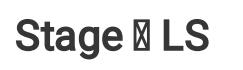 & Stage $\mathbb{L} \mathrm{LS}$ \\
\hline Number (male/female) & $30(17 / 13)$ & $64(28 / 36)$ & $58(14 / 44)$ \\
\hline Age, yr & $71.5 \pm 5.1^{\square}$ & $75.8 \pm 5.1^{\square}$ & $78.8 \pm 5.1^{\square}$ \\
\hline Body mass, $\mathrm{kg}$ & $55.5 \pm 8.8$ & $56.8 \pm 9.7$ & $54.2 \pm 9.5$ \\
\hline Height, cm & $156.5 \pm 8.7^{\square}$ & $154.8 \pm 8.6^{\square}$ & $150.2 \pm 7.8^{\square}$ \\
\hline Body mass index & $22.6 \pm 3.0$ & $23.6 \pm 2.9$ & $24.0 \pm 3.6$ \\
\hline 25-Question GLFS & $1.9 \pm 1.7^{\square}$ & $5.8 \pm 4.2^{\square}$ & $21.4 \pm 15.5^{\square}$ \\
\hline Two-step test & $1.47 \pm 0.09^{\square}$ & $1.31 \pm 0.11^{\square}$ & $1.14 \pm 0.15^{\square}$ \\
\hline Stand-up test & $5.3 \pm 0.5^{\square}$ & $4.0 \pm 0.8^{\square}$ & $3.2 \pm 1.1^{\square}$ \\
\hline \multicolumn{4}{|c|}{$\begin{array}{l}\text { Values are presented as the mean } \pm \text { standard deviation. Roman numerals in superscripts }(\mathbb{Q}-\mathbb{Q}) \text { next } t \\
\text { the data indicate the results of the post hoc analysis. The same numeral indicates no significant } \\
\text { difference between the two groups; different numerals indicate a significant difference between the } \\
\text { two groups }(p<0.05) \text {. }\end{array}$} \\
\hline \multicolumn{4}{|c|}{ LS = locomotive syndrome, GLFS = Geriatric Locomotive Function Scale. } \\
\hline
\end{tabular}

\section{STS Score}

During STS movement, similar curves for the VGRF, COP position, and trajectory were obtained for each group (Fig. 3). At the start of STS movement, the VGRF decreased because of hip flexion. Then, hip and 
knee extensions start and the VGRF reaches a peak value and oscillates around the body weight. Within 3 seconds from the start of the STS movement, the oscillation disappeared and the total COP distance was substantially stable (although not a plateau). Similar waveforms were observed among the groups, but differences were noted in the STS, speed, and balance scores (Table 2).

Table 2

STS Score, STS Power, TUG Time, and Handgrip Strength among LS Grades

\begin{tabular}{|c|c|c|c|}
\hline Measure & Non-LS & Stage $₫$ LS & 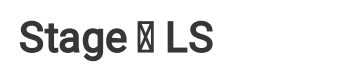 \\
\hline STS score, $(\mathrm{ms})^{-1}$ & $34.1 \pm 10.1^{\square}$ & $28.8 \pm 8.6^{\square}$ & $24.3 \pm 8.7^{\square}$ \\
\hline Speed score, $\mathrm{s}^{-1}$ & $9.5 \pm 2.3^{\square}$ & $8.5 \pm 2.2^{\square}$ & $6.9 \pm 1.7^{\square}$ \\
\hline Balance score, $\mathrm{m}^{-1}$ & $3.6 \pm 0.7$ & $3.4 \pm 0.8$ & $3.5 \pm 0.8$ \\
\hline Peak power, $\mathrm{W}$ & $391.7 \pm 104.5$ & $378.1 \pm 98.9$ & $369.2 \pm 90.7$ \\
\hline TUG time, $s$ & $5.3 \pm 0.8 \square$ & $6.4 \pm 1.0^{\square}$ & $7.6 \pm 1.5^{\square}$ \\
\hline Handgrip strength, $\mathrm{N}$ & $30.2 \pm 8.6^{\square}$ & $26.3 \pm 7.9^{\square}$ & $21.1 \pm 6.4^{\square}$ \\
\hline \multicolumn{4}{|c|}{$\begin{array}{l}\text { Values are presented as the mean } \pm \text { standard deviation. Roman numerals in superscripts }(\nabla-\nabla) \text { next to } \\
\text { the data indicate the results of the post hoc analysis. The same numeral indicates no significant } \\
\text { difference between the two groups; different numerals indicate a significant difference between the } \\
\text { two groups }(p<0.05) \text {. }\end{array}$} \\
\hline \multicolumn{4}{|c|}{ LS = locomotive syndrome; STS = sit to stand; TUG = timed up and go. } \\
\hline
\end{tabular}

The STS and speed scores could differentiate among the three groups (both $p<0.001)$. The STS score decreased significantly with the severity of LS. A significant difference in the speed score was noted between non-LS, stage $\otimes L S$, and stage $\otimes L S$. The speed score decreased with increasing LS grade. However, for the balance score, no significant differences were observed $(p=0.408)$.

\section{TUG Time, Handgrip Strength, and Peak Power}

The TUG time increased significantly according to the severity of LS. For handgrip strength, no significant difference was noted between non-LS and stage I LS or stage II LS. The peak power decreased when the LS grade increased. However, no significant differences were observed.

\section{Discussion}

Prevention of the development of LS and deterioration in motor performance are crucial to maintain the QOL in older adults. Simple indicators are needed to estimate individual physical performance and mobility for the early detection of LS. The STS score was developed to quantify STS movement based on the speed and balance parameters because STS movement changes over time as people grow older, resulting in a loss of balance control and muscle strength. This study demonstrated that the STS score decreases significantly according to the severity of LS in individuals older than 65 years. The STS score 
may be used to screen LS. We thus defined a "locomo-age" that describes an individual's age represented by their LS status. The locomo-age can be calculated from a regression equation between STS scores and age. The quadratic curve equation could be determined by a regression model based on the mean STS score and mean age in each of the seven 10-year age groups from 503 healthy individuals aged 2088 years (Appendix A) [6]. Using this equation, the individual's STS score can be converted to the locomoage. If the individual's locomo-age is higher than their age in years, then the individual would be at risk of developing LS. Thus, the locomo-age enables an individual to recognize their LS status more easily.

We used the STS score that was defined as a combination of the speed and balance scores, with the intention to quantify performance of STS movement using the VGRF and COP from the WBB. Our results showed significant decreases in the speed score with the development of LS. In contrast, the balance score could not differentiate the severity of LS. This finding implies that participants with a decline in motor performance must decrease their movement speed to maintain dynamic balance to accomplish STS movement more safely. These results emphasize the importance of combining both speed and balance parameters to evaluate the motor status. In fact, gait needs dynamic balance, and the gait speed decreases with age [32].

The TUG time was also good indicator to detect the LS status. This time seems to be a more significant parameter because the test includes walking, standing up, turning, stopping, and sitting down. However, the TUG test also needs a trained operator, such as a physiotherapist, and large space [33].

Although the handgrip strength served as an independent predictor to assess ADL performance, it is not a useful measurement to evaluate lower-extremity strength [34]. Our results further validate that the handgrip strength test is not suitable to detect early LS (stage $\rrbracket)$. Power has been demonstrated to be an early and potent marker of frailty [28]. In this study, the peak power could not detect LS in older adults. These results indicate that the simple muscle strength measurements may not be suitable to evaluate LS.

Several limitations of this study should be addressed. First, as we recruited participants from Japanese rojin clubs, these individuals may have been health conscious and may have had better motor abilities than the general population. It is necessary to conduct further studies that include members of the general population from defined regions. Second, a larger sample size is needed to analyze the cutoff value for LS.

\section{Conclusions}

Our results demonstrated a significant difference in the STS score across the range of severity of LS in participants older than 65 years. This simple and quantitative approach to quantify STS movement may be potentially useful as a tool to screen LS in older adult populations. In addition, we proposed the locomo-age to represent the individual's age for their LS status. Further studies are needed to elucidate the cutoff values of the STS score for the LS stages. 


\section{Abbreviations}

ADLs: activities of daily living; COP:center of pressure; GLFS:Geriatric Locomotive Function Scale; JOA:Japanese Orthopaedic Association; LS:locomotive syndrome; QOL:quality of life; STS:sit-to-stand; TUG:timed up and go; VGRF:vertical ground reaction force; WBB:Wii Balance Board.

\section{Declarations}

\section{Ethics approval and consent to participate}

Research protocol of this study followed the Declaration of Helsinki. This study was approved by the ethics committee of the University of Miyazaki (reference number: 2014-231). All the participants read and the understood the study protocol and a written informed consent was obtained prior to the experiment.

\section{Consent for publication}

Written informed consent was obtained to publish the images.

\section{Availability of data and material}

All data are available from the corresponding author upon request.

\section{Competing interest}

The authors declare that they have no competing interests. They declare that they have no financial or personal relationships with any other people or organizations that could have inappropriately influenced or biased this work.

\section{Funding}

This work was supported by JSPS KAKENHI Grant Number JP17K01362.

\section{Authors' Contribution}

GY designed the study and collected data, GY and NGP analyzed data and drafted the initial manuscript, HA helped data collection and data attraction, TT and ES developed the research question and guided the study. The authors read and approved the manuscript before submission.

\section{Acknowledgements}

We thank Y. Otsuka, S. Yamashita, H. Yoshikawa, K. Nakamura, M. Sugita, M. Nageyama, and M. Tsuruda for their support during data collection.

\section{References}


1. Granacher U, Muehlbauer T, Gollhofer A, Kressig RW, Zahner L: An intergenerational approach in the promotion of balance and strength for fall prevention - a mini-review. Gerontology 2011, 57(4):304315. doi:10.1159/000320250

2. Nakamura K: A "super-aged" society and the "locomotive syndrome". Journal of orthopaedic science: official journal of the Japanese Orthopaedic Association 2008, 13(1):1-2. doi:10.1007/s00776-007$1202-6$

3. Yoshimura N, Muraki S, Oka H, Tanaka S, Ogata T, Kawaguchi H, Akune T, Nakamura K: Association between new indices in the locomotive syndrome risk test and decline in mobility: third survey of the ROAD study. Journal of orthopaedic science: official journal of the Japanese Orthopaedic Association 2015. doi:10.1007/s00776-015-0741-5

4. Ogata T, Muranaga S, Ishibashi $\mathrm{H}$, Ohe T, Izumida R, Yoshimura N, Iwaya T, Nakamura K: Development of a screening program to assess motor function in the adult population: a crosssectional observational study. Journal of orthopaedic science: official journal of the Japanese Orthopaedic Association 2015, 20(5):888-895. doi:10.1007/s00776-015-0737-1

5. Kobayashi T, Morimoto T, Shimanoe C, Ono R, Otani K, Mawatari M: Development of a simple screening tool based on the 5-question geriatric locomotive function scale for locomotive syndrome. Journal of orthopaedic science: official journal of the Japanese Orthopaedic Association 2021. doi:10.1016/j.jos.2021.05.001

6. Yamako G, Chosa E, Totoribe K, Fukao Y, Deng G: Quantification of the sit-to-stand movement for monitoring age-related motor deterioration using the Nintendo Wii Balance Board. PloS one 2017, 12(11):e0188165. doi:10.1371/journal.pone.0188165

7. Tinetti ME, Speechley M, Ginter SF: Risk factors for falls among elderly persons living in the community. The New England journal of medicine 1988, 319(26):1701-1707. doi:10.1056/NEJM198812293192604

8. Cummings SR, Nevitt MC, Browner WS, Stone K, Fox KM, Ensrud KE, Cauley J, Black D, Vogt TM: Risk factors for hip fracture in white women. Study of Osteoporotic Fractures Research Group. The New England journal of medicine 1995, 332(12):767-773. doi:10.1056/NEJM199503233321202

9. Janssen WG, Bussmann HB, Stam HJ: Determinants of the sit-to-stand movement: a review. Physical therapy 2002, 82(9):866-879.

10. Riley PO, Schenkman ML, Mann RW, Hodge WA: Mechanics of a constrained chair-rise. Journal of biomechanics 1991, 24(1):77-85.

11. Rodosky MW, Andriacchi TP, Andersson GB: The influence of chair height on lower limb mechanics during rising. Journal of orthopaedic research: official publication of the Orthopaedic Research Society 1989, 7(2):266-271. doi:10.1002/jor.1100070215

12. Hsu WL, Chou LS, Woollacott M: Age-related changes in joint coordination during balance recovery. Age (Dordrecht, Netherlands) 2013, 35(4):1299-1309. doi:10.1007/s11357-012-9422-x

13. Tsai YC, Hsieh LF, Yang S: Age-related changes in posture response under a continuous and unexpected perturbation. Journal of biomechanics 2014, 47(2):482-490. 
doi:10.1016/j.jbiomech.2013.10.047

14. Yamako G, Deng G, Totoribe K, Chosa E: A novel protocol to test age-related decreases in sit-to-stand movement abilities in healthy subjects. Journal of orthopaedic science: official journal of the Japanese Orthopaedic Association 2016, 21(4):517-523. doi:10.1016/j.jos.2016.04.006

15. Papa E, Cappozzo A: Sit-to-stand motor strategies investigated in able-bodied young and elderly subjects. Journal of biomechanics 2000,33(9):1113-1122.

16. Hughes MA, Myers BS, Schenkman ML: The role of strength in rising from a chair in the functionally impaired elderly. Journal of biomechanics 1996, 29(12):1509-1513.

17. Mazza C, Zok M, Della Croce U: Sequencing sit-to-stand and upright posture for mobility limitation assessment: determination of the timing of the task phases from force platform data. Gait \& posture 2005, 21(4):425-431. doi:10.1016/j.gaitpost.2004.05.006

18. Hesse S, Schauer M, Malezic M, Jahnke M, Mauritz KH: Quantitative analysis of rising from a chair in healthy and hemiparetic subjects. Scandinavian journal of rehabilitation medicine 1994, 26(3):161166.

19. Nakamura K, Ogata T: Locomotive Syndrome: Definition and Management. Clin Rev Bone Miner Metab 2016, 14:56-67. doi:10.1007/s12018-016-9208-2

20. Ishibashi H: Locomotive syndrome in Japan. Osteoporos Sarcopenia 2018, 4(3):86-94. doi:10.1016/j.afos.2018.09.004

21. Bartlett HL, Ting LH, Bingham JT: Accuracy of force and center of pressure measures of the Wii Balance Board. Gait \& posture 2014, 39(1):224-228. doi:10.1016/j.gaitpost.2013.07.010

22. Clark RA, Bryant AL, Pua Y, McCrory P, Bennell K, Hunt M: Validity and reliability of the Nintendo Wii Balance Board for assessment of standing balance. Gait \& posture 2010, 31(3):307-310. doi:10.1016/j.gaitpost.2009.11.012

23. Huurnink A, Fransz DP, Kingma I, van Dieen JH: Comparison of a laboratory grade force platform with a Nintendo Wii Balance Board on measurement of postural control in single-leg stance balance tasks. Journal of biomechanics 2013, 46(7):1392-1395. doi:10.1016/j.jbiomech.2013.02.018

24. Abujaber S, Gillispie G, Marmon A, Zeni J, Jr.: Validity of the Nintendo Wii Balance Board to assess weight bearing asymmetry during sit-to-stand and return-to-sit task. Gait \& posture 2015, 41(2):676682. doi:10.1016/j.gaitpost.2015.01.023

25. Young W, Ferguson S, Brault S, Craig C: Assessing and training standing balance in older adults: a novel approach using the 'Nintendo Wii' Balance Board. Gait \& posture 2011, 33(2):303-305. doi:10.1016/j.gaitpost.2010.10.089

26. Goble DJ, Cone BL, Fling BW: Using the Wii Fit as a tool for balance assessment and neurorehabilitation: the first half decade of "Wii-search". J Neuroeng Rehabil 2014, 11:12. doi:10.1186/1743-0003-11-12

27. Skelton DA, Greig CA, Davies JM, Young A: Strength, power and related functional ability of healthy people aged 65-89 years. Age Ageing 1994, 23(5):371-377. doi:10.1093/ageing/23.5.371 
28. Lindemann U, Claus H, Stuber M, Augat P, Muche R, Nikolaus T, Becker C: Measuring power during the sit-to-stand transfer. Eur J Appl Physiol 2003, 89(5):466-470. doi:10.1007/s00421-003-0837-z

29. Zijlstra W, Bisseling RW, Schlumbohm S, Baldus H: A body-fixed-sensor-based analysis of power during sit-to-stand movements. Gait \& posture 2010, 31(2):272-278.

doi:10.1016/j.gaitpost.2009.11.003

30. Podsiadlo D, Richardson S: The timed "Up \& Go": a test of basic functional mobility for frail elderly persons. Journal of the American Geriatrics Society 1991, 39(2):142-148.

31. Al Snih S, Markides KS, Ottenbacher KJ, Raji MA: Hand grip strength and incident ADL disability in elderly Mexican Americans over a seven-year period. Aging Clin Exp Res 2004, 16(6):481-486. doi:10.1007/BF03327406

32. Kyrdalen IL, Thingstad P, Sandvik L, Ormstad H: Associations between gait speed and well-known fall risk factors among community-dwelling older adults. Physiother Res Int 2019, 24(1):e1743. doi:10.1002/pri.1743

33. Fudickar S, Hellmers S, Lau S, Diekmann R, Bauer JM, Hein A: Measurement System for Unsupervised Standardized Assessment of Timed "Up \& Go" and Five Times Sit to Stand Test in the Community-A Validity Study. Sensors (Basel) 2020, 20(10). doi:10.3390/s20102824

34. Rodacki ALF, Boneti Moreira N, Pitta A, Wolf R, Melo Filho J, Rodacki CLN, Pereira G: Is Handgrip Strength a Useful Measure to Evaluate Lower Limb Strength and Functional Performance in Older Women? Clin Interv Aging 2020, 15:1045-1056. doi:10.2147/CIA.S253262

\section{Figures}




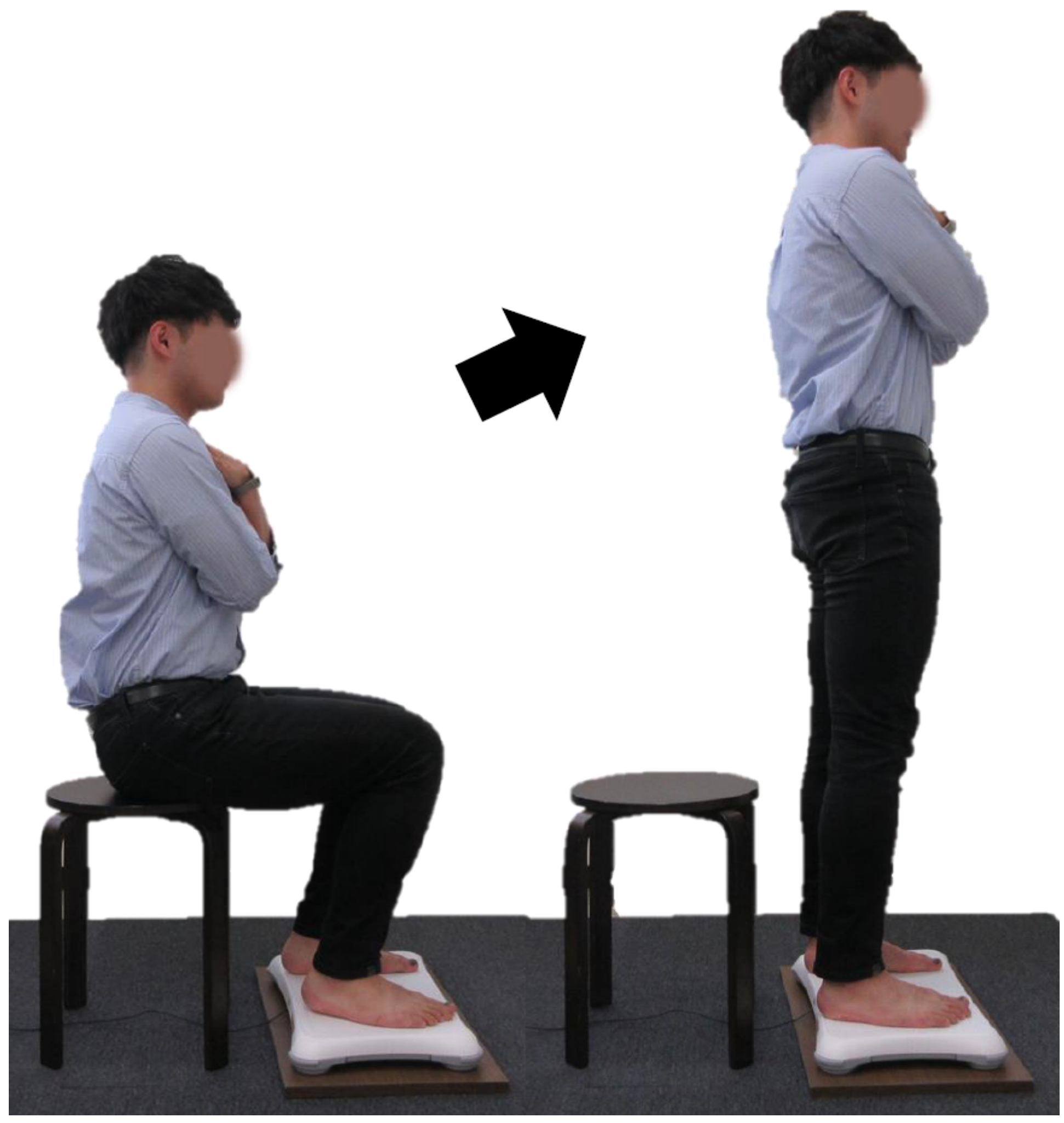

Figure 1

Sit-to-stand (STS) movement test. The Wii Balance Board is placed under the feet. 


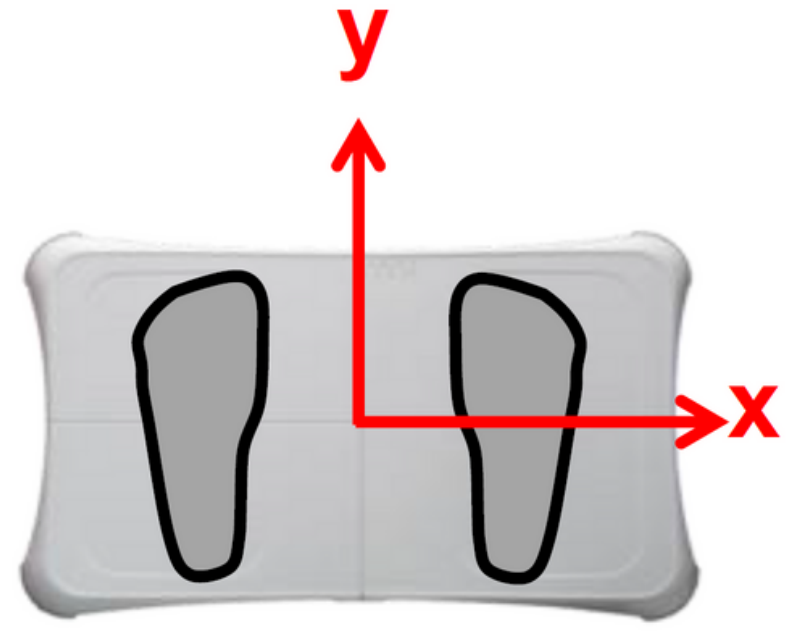

Balance score: 3.31

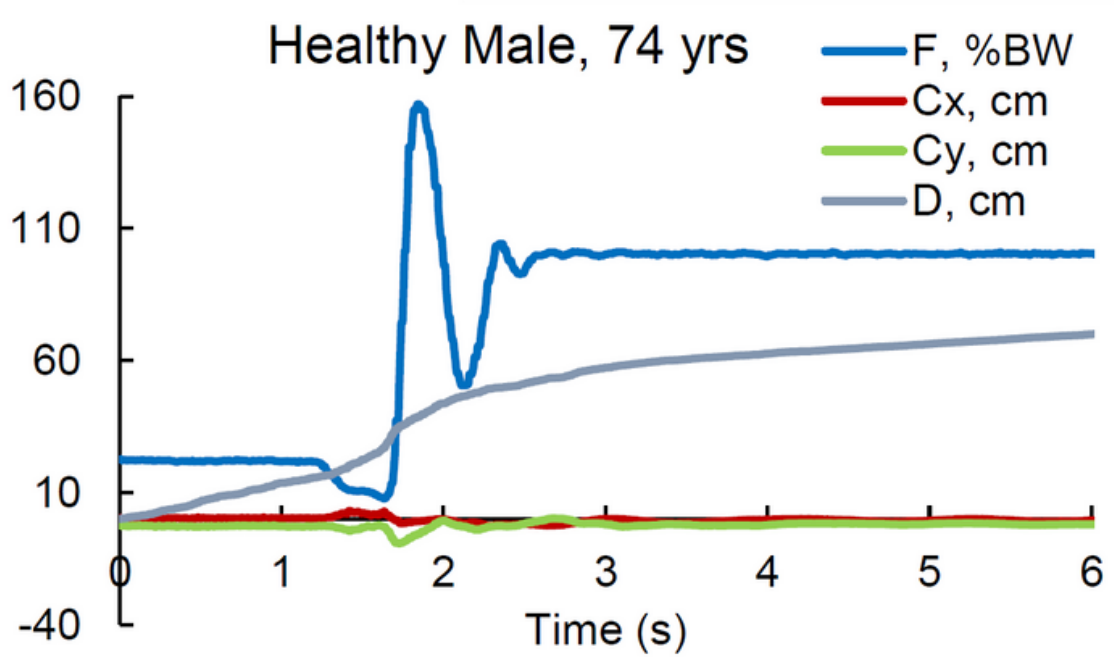

Figure 2

(a) Coordinate system of the Wii Balance Board and (b) a typical graph of the sit-to-stand (STS) test performed by a healthy person. The vertical ground reaction force $(F)$ normalized by the body weight (BW) and center of pressure (COP) in the $\mathrm{X}(\mathrm{Cx})$ and $\mathrm{Y}(\mathrm{Cy})$ directions are plotted in the graph.

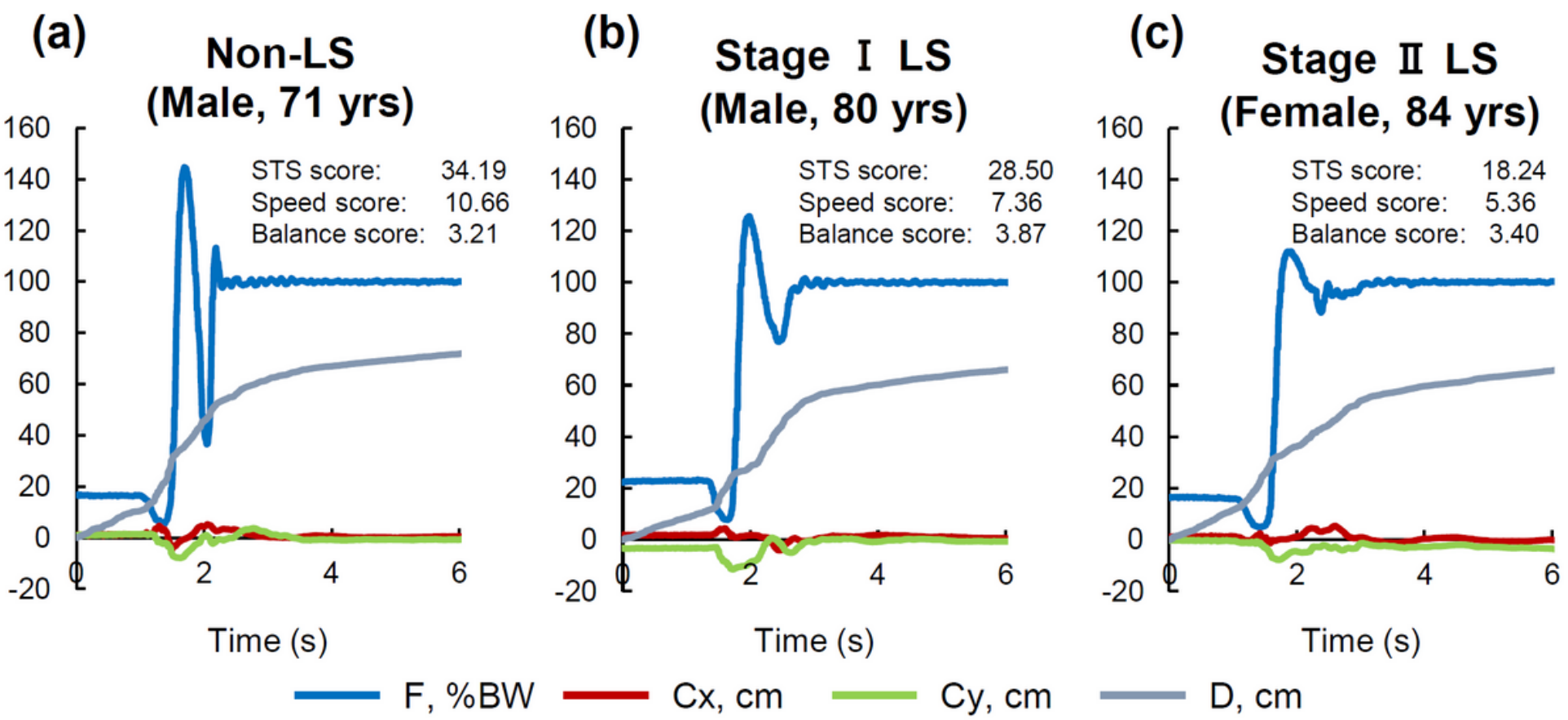

Figure 3

Typical graph patterns of the vertical ground reaction force and total center of pressure (COP) for each locomotive syndrome stage. F, vertical ground reaction force; $\mathrm{Cx}$ and $\mathrm{Cy}, \mathrm{COP}$ trajectories in the Wii Balance Board; D, trajectory distance. 


\section{Supplementary Files}

This is a list of supplementary files associated with this preprint. Click to download.

- AppendixA.docx 